\title{
COMPARISON OF PADC NEUTRON DETECTORS FROM DIFFERENT SUPPLIERS
}

\author{
M. Caresana ${ }^{1, *}$, A. Parravicini ${ }^{2}$, S. Coria ${ }^{2}$, A. Stabilini ${ }^{1,3}$, F. Assenmacher ${ }^{3}$ and E.G. Yukihara $^{3,4}$ \\ ${ }^{1}$ Politecnico di Milano, Dipartimento di Energia, via la Masa 34, 20156 Milan, Italy \\ ${ }^{2}$ MI.AM srl, via De Amicis 5, Fabbiano, Italy \\ ${ }^{3}$ Paul Scherrer Institute, Department of Radiation Safety and Security, 5232 Villigen PSI, Switzerland \\ ${ }^{4}$ Oklahoma State University, Physics Department, Stillwater, OK 74078, USA \\ *Corresponding author: marco.caresana@polimi.it
}

\begin{abstract}
The objective of this study was to compare poly-allyl diglycol carbonate (PADC) track detectors from different suppliers for linear energy transfer (LET) spectrometry and neutron dosimetry. PADCs are commonly used for passive personal neutron dosimetry, where a common approach is to couple the PADC with a plastic radiator to generate secondary charged particles. The neutron dose can be estimated using the track density or the average LET of the secondary particles. The characterisation of PADC in terms of LET spectrometric capability and neutron sensitivity was previously performed using PADC from Intercast S.r.l., Parma, Italy, but this company stopped the production. Since it is common experience that material from different suppliers can have different properties, it became necessary to perform a comparison of PADCs from different suppliers with the Intercast material. The study permits to compare the reading procedures used at Politecnico di Milano and at the Paul Scherrer Institute.
\end{abstract}

\section{INTRODUCTION}

Poly-allyl diglycol carbonate (PADC)-based neutron dosimetry is one of the most used techniques for passive personal neutron dosimetry. One approach consists in coupling the PADC to a plastic radiator and counting the neutron-induced tracks due to secondary particles created by neutron interaction with the radiator and the PADC itself, mainly protons in case of neutron energy below $\sim 10 \mathrm{MeV}$. For higher neutron energy, ${ }^{2} \mathrm{H},{ }^{3} \mathrm{H}$ and alpha particles can be produced via inelastic scattering. Alternatively, the tracks can be analysed to calculate the linear energy transfer (LET) spectrum of the secondary particles in the detector, from which a dose can be obtained by weighting the energy deposited by each particle with the LET-dependent quality factor ${ }^{(1)}$. The LET measured by the PADC is averaged over the portion of the particle path defined by the product of the track etch rate by the etching time. In the article, this quantity is referred as LETnc to underline the difference from the definition of the LET.

A previous study ${ }^{(1,3)}$ characterised PADCs from Intercast S.r.1. (Parma, Italy) in terms of neutron sensitivity and LETnc spectrometry capabilities, but the company stopped the production of PADC as track detectors. This raised the need to evaluate PADCs from other suppliers, which can have different sensitivities due to variations in preparation, casting and chemical additives.

The objective of this work was to perform a study of PADCs from different suppliers (Table 1) for neutron dosimetry and LETnc spectrometry application. These
PADCs were compared to the Intercast material in terms of: (1) bulk etch rate, estimated with the fission fragment technique ${ }^{(2)},(2)$ average LETnc distribution of monochromatic alpha particles ${ }^{(3)}$, (3) background track density and (4) sensitivity to neutrons from an ${ }^{241} \mathrm{Am}-\mathrm{Be}$ source. The study also offered an opportunity to compare the reading systems and etching procedures used at Politecnico di Milano (POLIMI) and at Paul Scherrer Institute (PSI).

\section{METHODOLOGY}

The PADC detectors investigated (Table 1) were $2.5 \mathrm{~cm} \times 2.5 \mathrm{~cm} \times 1.5 \mathrm{~mm}$, except for the RTP-USA, whose thickness was $1 \mathrm{~mm}$. To determine the bulk etch rate and the mean LET spectrum of alpha particles, six detectors from each supplier were irradiated with an electroplated ${ }^{252} \mathrm{Cf}$ source, and chemically etched at MI.AM with an aqueous solution of $\mathrm{NaOH} 6.25 \mathrm{M}$ at $98^{\circ} \mathrm{C}$ for 40,60 and $90 \mathrm{~min}$ (two detectors each). For each supplier and etching condition, two unirradiated detectors were also used to estimate the background signal. To estimate the neutron response, 12 detectors of every supplier were coupled with a $2 \mathrm{~mm}$ thick polyethylene radiator and exposed on a phantom to the $3 \mathrm{mSv} \mathrm{AmBe}$ reference neutron source at $\mathrm{PSI}^{(4)}$. From these detectors, six were etched according to the standard etching procedures at PSI $\left(2 \mathrm{~h} \text { and } 50 \mathrm{~min} \text { at } 85^{\circ} \mathrm{C}\right)^{(5)}$ and six according to the MI.AM etching procedure $(90 \mathrm{~min}$ at $98^{\circ} \mathrm{C}$ ). 
COMPARISON OF PADC NEUTRON DETECTORS

Table 1. PADC detector used in this study. For RTP the percentage refers to different DOP (di-octyl phthalate) concentration.

\begin{tabular}{ll} 
Supplier & Detector name \\
\hline Track Analysis System Ltd. & TASTRAK \\
Intercast Europe S.r.l. & Intercast \\
Chiyoda Technol Corporation & Chiyoda \\
RTP-USA & RTP 0.1\% \\
& RTP 0.5\% \\
& RTP 1\% \\
Mi-Net Technology Ltd. & Mi-Net
\end{tabular}

The bulk etch rate $\left(V_{b}\right)$ estimation was obtained using the fission fragment technique ${ }^{(2)}$, where, from the minor axis measurement of the track openings generated by heavy fragments and knowing the etching time $(t)$, the bulk etch rate can be estimated relaying on the approximated equation:

$$
V_{b} \cong d / 2 t
$$

The alpha particles LETnc distribution was accomplished by acquiring the major and minor axis of the etched tracks ${ }^{(3)}$. From these values the reduced track etch rate ratio $(V)$ can be obtained for each track and used to find the LETnc of each particle, relying on parametric correlations between the reduced track etch rate ratio $V$ and the average particle LETnc. The same correlation was used for each detector listed in Table 1.

The track density analysis of unirradiated and neutron irradiated detectors was achieved relying on the TASLIMAGE ${ }^{\mathrm{TM}^{(5)}}$ and Politrack ${ }^{\mathrm{TM}(3)}$ embedded algorithms, adjusting the filtering parameters when necessary. In addition, Politrack ${ }^{\mathrm{TM}}$ allowed the dose assessment by embedding the LETnc spectrometry analysis in the evaluation software ${ }^{(1)}$.

\section{RESULTS AND DISCUSSION}

\section{Bulk etch rate}

The results on the $V_{b}$ determination are shown in Figure 1. The graph shows the removed layer as a function of the etching time, confirming the linear trend expected for each material (excluding a nonlinear region in the beginning of the etch or $\sim 25$ $\left.\min ^{(6,7)}\right)$. The TASTRAK and RTP $0.1 \%$ are the 'softest' tested materials, with a $V_{b}$ of 14 and $12.8 \mu \mathrm{m} / \mathrm{h}$, respectively (Table 2), i.e. are more deeply etched by the chemical solution in comparison to the other materials under the same etching conditions. On the other hand, Chiyoda and Intercast are the 'hardest' ones having $V_{b}=9.2 \mu \mathrm{m} / \mathrm{h}$. The removed layer measured for the PSI etching and the MI.AM

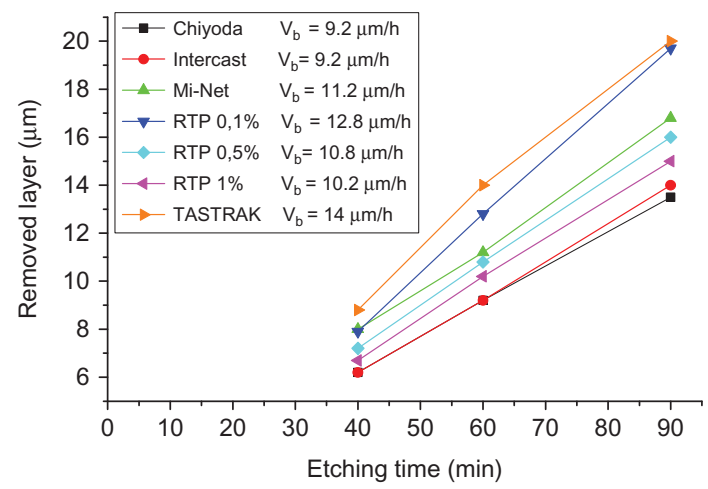

Figure 1. Removed layer versus etch duration, showing the bulk etch rate $\left(V_{b}\right)$.

etching at $60 \mathrm{~min}$ is the same. For this reason, they are considered equivalent.

\section{LETnc distribution of alpha particles}

Figure 2 compares the LETnc spectra of ${ }^{252} \mathrm{Cf}$ alpha particles $(6.1 \mathrm{MeV})$ obtained using the $98^{\circ} \mathrm{C} / 60 \mathrm{~min}$ etching. Each material showed a clear distribution centred on the theoretical value of $120 \mathrm{keV} / \mu \mathrm{m}^{(2)}$. The achieved resolution of the materials for LETnc spectrometry can be inferred from the FWHM (Table 2), which shows good values $(<21 \mathrm{keV} / \mu \mathrm{m})$ for RTP $0.5 \%$, RTP $1 \%$, Intercast and TASTRAK.

\section{Background track density}

Figure 3 presents the results of unirradiated detectors, at different etching times, evaluated by Politrack $^{\mathrm{TM}}$ system and TASLIMAGE ${ }^{\mathrm{TM}}$. For both systems the background track density of the RTP detectors is fairly high, whereas TASTRAK, Mi-Net and Chiyoda detectors show a lower background signal. TASTRAK detectors evaluated using the TASLIMAGE ${ }^{\mathrm{TM}}$, showed low background, consistent with the fact that the track analysis algorithm is optimised for this material. The background signal of the Intercast detectors is relatively high because the detectors are from an 8-year-old batch and have accumulated tracks from cosmic ray background whose increase was observed during time. The TASLIMAGE ${ }^{\mathrm{TM}}$ data referring to $90 \mathrm{~min}$ etching are missing, because the system was not able to read these long etched detectors within the current analysis parameters. Overall, for 40 and 60 min etching the two reading systems gave comparable results, except for RTP 0.5 and $1 \%$ where 60 min etching shows an unexpected important difference.

Figure 4 shows the average track density values of unirradiated detectors etched for $90 \mathrm{~min}$ at $98^{\circ} \mathrm{C}$ and 
M. CARESANA ET AL.

Table 2. Comparison of different detectors for LETnc measurement. The theoretical value of the LETnc is $120 \mathrm{keV} / \mu \mathrm{m}$.

\begin{tabular}{lccc}
\hline Supplier & $\begin{array}{c}\text { Bulk etch rate } \\
\left(V_{b}\right)(\mu \mathrm{m} / \mathrm{h})\end{array}$ & $\begin{array}{l}\text { Mean LET } \\
(\mathrm{keV} / \mu \mathrm{m})\end{array}$ & $\begin{array}{l}\text { FWHM } \\
(\mathrm{keV} / \mu \mathrm{m})\end{array}$ \\
\hline RTP 0.1\% & 12.8 & 120.3 & 30.9 \\
RTP 0.5\% & 10.8 & 120.7 & 15.3 \\
RTP 1\% & 10.2 & 119.2 & 20.6 \\
Intercast & 9.2 & 120.3 & 18.2 \\
TASTRAK & 14 & 121.0 & 20.8 \\
Mi-Net & 11.2 & 119.2 & 48.4 \\
Chiyoda & 9.2 & 123.7 & 25.7 \\
\hline
\end{tabular}

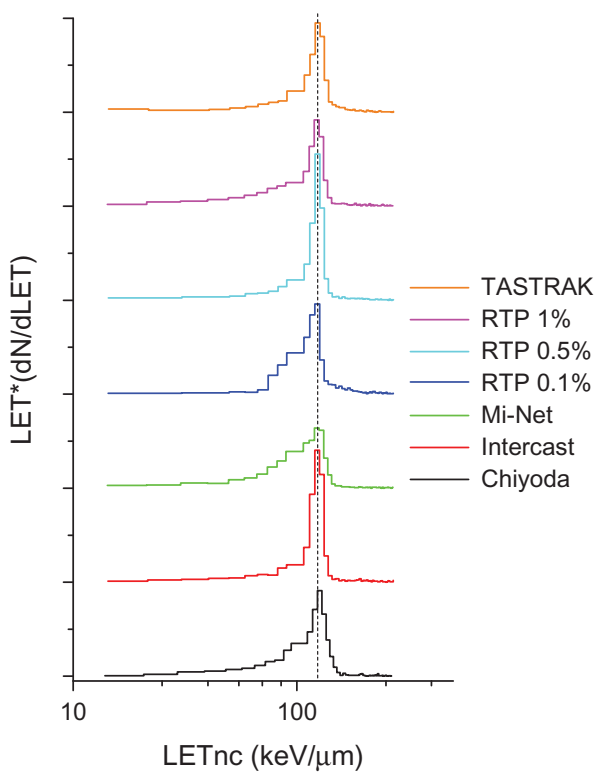

Figure 2. LETnc spectra of detectors from different suppliers irradiated with electroplated ${ }^{252} \mathrm{Cf}$ and etched by $6.25 \mathrm{M}$ of $\mathrm{NaOH}$ at $98^{\circ} \mathrm{C}$ for $60 \mathrm{~min}$.

the dose for the same detectors calculated using the LETnc spectrum. RTPs are characterised by a rather high spread of background signal, deriving from an important variability within detectors. Using the LETnc spectrum, the background signal can be decreased in certain cases. In fact, considering RTP $0.1 \%$ and Intercast, the track density of the RTP $0.1 \%$ is higher than the Intercast one, but the estimated background doses are lower. This can be explained looking at the inset graph in Figure 4, where the LETnc distribution is shown for RTP $0.1 \%$ and for Intercast. In the case of RTP $0.1 \%$ the signal is mainly due to very small tracks, originating from fabrication defects, which are similar to the

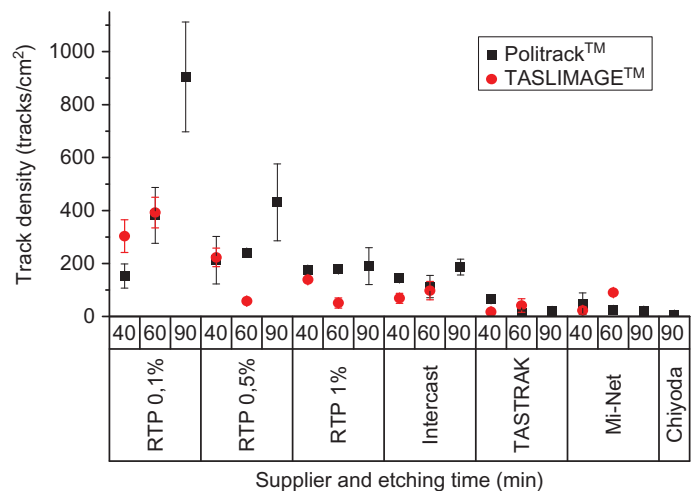

Figure 3. Track density of unirradiated detectors versus etching time for analysis using Politrack ${ }^{\mathrm{TM}}$ and TASLIMAGE $E^{\mathrm{TM}}$. Chiyoda PADC have been etched at 90 min only because a limited number of detector was available.

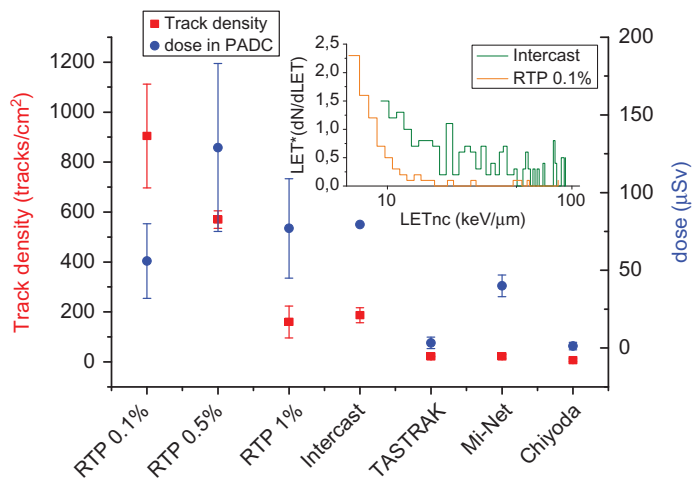

Figure 4. (a) Average track densities and calculated doses for unirradiated detectors. (b) Examples of LETnc distribution of unirradiated detectors from Intercast and RTP $0.1 \%$. (90 $\mathrm{min} / 98^{\circ} \mathrm{C}$ etching.)

tracks from low-LET particles, thus not contributing much to the dose. Conversely the background signal from Intercast, coming from cosmic radiation, produces wider tracks to which higher LETnc values are associated, hence, yielding a higher dose background.

\section{Response to neutrons}

Figure 5 presents the sensitivity (background subtracted) of the various PADCs after $3 \mathrm{mSv}$ neutron irradiation. The graph compares data from the PSI and POLIMI readers, with different etching procedures. The PSI etching removed a layer numerically equal to the $V_{b}$ reported in Table 2 and generally resulted in higher sensitivity. The sensitivities 


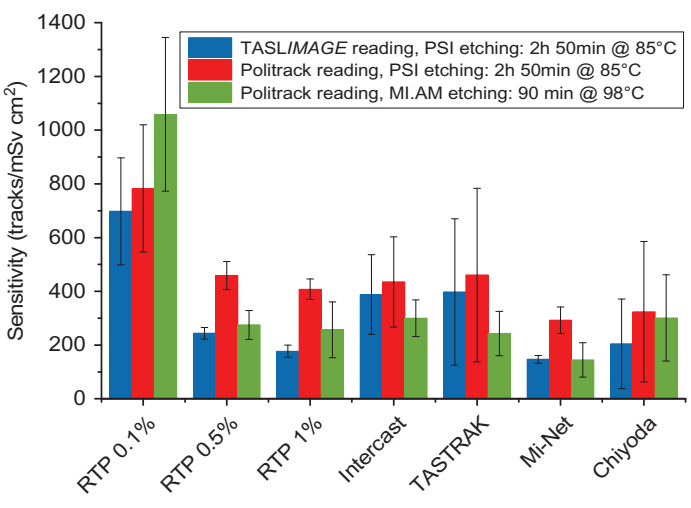

Figure 5. Neutron sensitivity of detectors from different suppliers, readout systems, and etching procedures. The thickness of the removed layer for PSI etching is numerically equal to the $V_{b}$ reported in Table 2 while the one for MI.AM etching is 1.5 times higher.

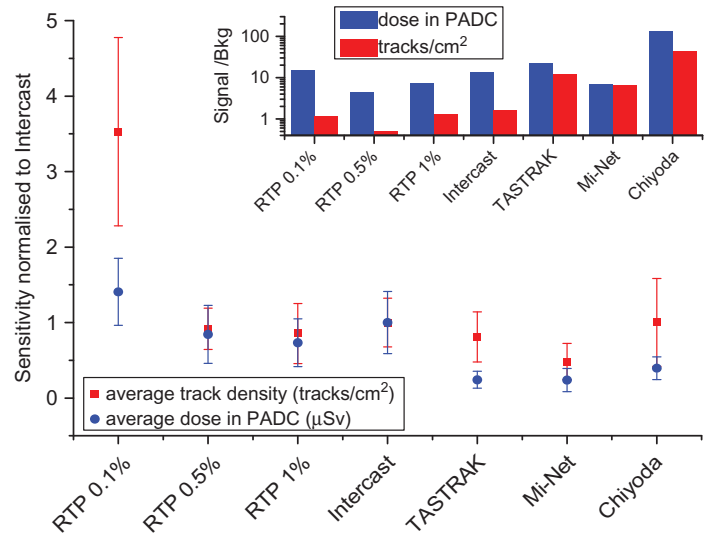

Figure 6. Sensitivity in terms of track density and average dose in PADC detectors irradiated with $3 \mathrm{mSv}$ with the AmBe neutron source, normalised to the Intercast values. The inset graph shows the Signal/Bkg of irradiated detectors grouped by supplier, calculated based on track densities or LETnc spectrum (dose). Readout system Politrack.

measured by Politrack ${ }^{\mathrm{TM}}$ were also typically higher: this is because the Politrack ${ }^{\mathrm{TM}}$ operates at higher magnification, thus the filtering algorithm can better resolve the contour of each track ending up in a decreased number of rejected tracks. Despite the high background, RTP $0.1 \%$ seems to have a higher response to neutrons.

Figure 6 compares the response of neutron irradiated detectors, calculated using the track density or the LETnc spectrum, normalised to the Intercast response.

The inset shows the Signal/Bkg ratio for each supplier, also calculated using the track density or the
LETnc spectrum. Although the RTP detectors have higher response, the background is also high, therefore, resulting in poor signal to background ratio, except for the RTP $0.1 \%$ (dose). On the other hand, TASTRAK and Chiyoda showed better Signal/Bkg ratio, despite lower sensitivity.

\section{CONCLUSIONS}

PADCs from different suppliers were compared with the performances of INTERCAST, which is no more produced. The study allowed to compare the etching procedures and the PADC readers used by PSI and POLIMI. The results show that PSI etching offers better performances for neutron dosimetry in terms of track density. For both scanning systems the track densities were similar in the case of RTP $0.1 \%$, Intercast, TASTRAK and Chiyoda. The RTP 0.1\% shows a very high sensitivity, almost three times higher than the average of the other detectors. On the other hand, RTPs have a very high background, resulting in a high limit of detection. Dose estimations based on LETnc spectrum could reduce background in particular for RTP $0.1 \%$. Signal/Bkg in terms of track density is rather poor for RTP and good for TASL, Mi-Net and Chiyoda, whereas, in terms of dose, also RTP $0.1 \%$ offers a satisfactory Signal/Bkg ratio. All the detectors are in principle fit for neutron dosimetry, with pro (high sensitivity) and cons (high background). This study helps to characterise the detectors but cannot identify the best performing one because it is limited to irradiation with an $\mathrm{Am}-\mathrm{Be}$ source, without taking into account the spectral sensitivity. Effects like ageing and fading are also neglected. Moreover, the statistic is poor because of the low number of detectors available. We foresee a more in-depth characterisation, with a larger statistical sampling.

\section{ACKNOWLEDGEMENTS}

The authors thank Kilian Meier and Helga Schröter for the detector irradiation and etching.

\section{FUNDING}

This work was partly supported by the Swiss Nuclear Safety Inspectorate (Contract no. H-101196).

\section{REFERENCES}

1. Caresana, M. et al. Evaluation of a personal and environmental dosemeter based on CR-39 track detectors in quasi-monoenergetic neutron fields. Radiat. Prot. Dosim. 161(1-4), 100-103 (2014).

2. Nikezic, D. and Yu, K. Formation and growth of tracks in nuclear track materials. Mater. Sci. Eng. R 46(3-5), 51-123 (2004). 


\section{CARESANA ET AL.}

3. Caresana, M. et al. Determination of LET in PADC detectors through the measurement of track parameters. Nucl. Instrum. Methods A 683, 8-15 (2012).

4. Hoedlmoser, H. et al. Characteristics of the neutron irradiation facilities of the PSI calibration laboratory. AIP Conf. Proc. 1412, 385-392 (2011).

5. Boschung, M. et al. Field calibration and comparison of personal neutron dosemeter designs based on CR-39 for the use around high-energy accelerators. Radiat. Meas. 43(2-6), 1081-1084 (2008).

6. Leonardi, F. et al. An extended study of the etching characteristics of CR-39 detectors. Radiat. Meas. 44(9-10), 787-790 (2009).

7. Yamauchi, T. et al. Surface layer in CR-39 plastic track detector where the bulk etch rate is enhanced. Radiat. Meas. 37(2), 119-125 (2003). 